\title{
Infinite Potential Barrier and Hydrostatic Pressure Effects on Impurity-Related Optical Absorption Spectra in GaAs Double Quantum Wells
}

\author{
N. Raigoza, A. L. Morales, and C. A. Duque \\ Instituto de Física, Universidad de Antioquia, AA 1226, Medellín, Colombia
}

Received on 4 April, 2005

\begin{abstract}
Using the effective-mass approximation and the variational method, we have calculated the effects of hydrostatic pressure on the donor- and acceptor-related optical absorption spectra in symmetrical GaAs double quantum well structures. A central finite potential barrier and two infinite external barriers constitute the profile of the potential barrier considered for the wells. Our results are presented as a function of the well and barrier widths and hydrostatic pressure. For the pressure dependence we consider the $\Gamma-X$ mixing in the central barrier layer. For symmetrical and infinite-external-barrier quantum wells, and depending on the sizes of the structure and the hydrostatic pressure, the donor-related spectra show three special structures, whereas for the acceptor one only two structures appear

Keywords: GaAs Double Quantum Wells; Effective-mass approximation; Hydrostatic pressure
\end{abstract}

\section{INTRODUCTION}

In the last years, the study of hydrostatic pressure on the optical properties of quantum confined structures have been widely investigated [1-7]. Even since the advent of layered semiconductor structures, one of the main research interests has been the study of the optical properties of semiconductor quantum wells $(\mathrm{QW})$. Not only have the optical properties turned out to be of significant use for technological applications, but they also provide important information about the fundamental behavior of electronic properties in an environment of reduced dimensionality. The study of hydrostatic pressure influence on optical properties of low-dimensional systems is important from both fundamental and theoretical points of view. It is well known that hydrostatic pressure can be used to modify the band structure of semiconductors. In some cases, the absolute conduction band minimum may shift from one symmetry point to another. For example, at hydrostatic pressures above $40 \mathrm{kbar}$, GaAs becomes an indirect band-gap semiconductor [3]. The use of low-temperature photoluminescence measurements made it possible to study the electronic and optical properties of microstructure semiconductor under high hydrostatic pressure. Some authors observed the Raman spectra and optical phonon energies in bulk GaAlAs material under pressure [4]. Bauer and Ando [1] have reported the pressure, magnetic field, and electric field effects on the excitonic systems in GaAs quantum wells. The effects of the $\Gamma-X$ crossover pressure at low-temperatures on the donor binding energies in $\mathrm{QW}$ heterostructures have been studied by Elabsy [5]. The combined effect of hydrostatic pressure and applied electric field on the binding energy and density of shallow impurity states (DOIS) in symmetrical and asymmetrical GaAs- $\mathrm{Ga}_{1-x} \mathrm{Al}_{x}$ As double quantum wells (DQW) have also been calculated $[6,7]$.

In the present work we make theoretical development about the effects of hydrostatic pressure on the binding energy and the optical-absorption spectra associated with shallow-impurities (donor and acceptor) in symmetrical GaAs- $\mathrm{Ga}_{0.7} \mathrm{Al}_{0.3} \mathrm{As} \mathrm{DQW}$, by using the effective mass approximation and the variational method. The optical- absorption spectra is calculated for transitions from the first valence subband to the donor-impurity band and from the acceptor-impurity band to the first conduction subband, considering that the impurities are evenly distributed in the region of the central barrier and the two QWs. The paper is organized as follows: in section 2 we present our theoretical model, in section 3 our results and discussion, and finally in section 4 the conclusions are presented.

\section{THEORETICAL FRAMEWORK}

In the effective-mass approximation, the Hamiltonian for a hydrogenic shallow impurity in a GaAs- $\mathrm{Ga}_{0.7} \mathrm{Al}_{0.3} \mathrm{As} \mathrm{DQW}$ under the effect of hydrostatic pressure $(P)$, at low temperature $(T)$ is given by

$$
\begin{array}{r}
H=-\frac{\hbar^{2}}{2} \nabla \cdot\left(\frac{1}{m_{w c, b c}^{*}(P, T)} \nabla\right)+V_{c}(z, P) \\
-\frac{e^{2}}{\varepsilon_{w, b}^{*}(P, T) r},
\end{array}
$$

where $r$ is the carrier-impurity distance. Subscripts $w$ and $b$ stand for the well and barrier layer materials, respectively. $m_{w c, b c}^{*}$ are the well and barrier materials conduction effectivemasses, and $\varepsilon_{w, b}^{*}$ are the corresponding static dielectric constants $[5,8-10]$. In the well region we have used for the donorelectron effective mass and the static dielectric constant, respectively,

$$
\frac{m_{w c}^{*}(P, T)}{m_{0}}=\left[1+E_{P}^{\Gamma}\left(\frac{2}{E_{g}^{\Gamma}}+\frac{1}{E_{g}^{\Gamma}+\Delta_{0}}\right)\right]^{-1}
$$

and

$$
\varepsilon_{w}^{*}(P, T)=\varepsilon_{0} e^{\delta_{1}\left(T-T_{0}\right)} e^{-\delta_{2} P},
$$

where $m_{0}$ is the free electron mass, $E_{g}^{\Gamma}=E_{g}^{\Gamma}(P, T)$ is the pressure and temperature dependent GaAs band gap, 
$E_{P}^{\Gamma}=7.51 \mathrm{eV}, \Delta_{0}=0.341 \mathrm{eV}, \varepsilon_{0}=12.74, \delta_{1}=9.4 \times 10^{-5} \mathrm{~K}^{-1}$, $\delta_{2}=1.67 \times 10^{-3} \mathrm{kbar}^{-1}$, and $T_{0}=75.6 \mathrm{~K}$. For the barrier parameters $\left(m_{b c}^{*}\right.$ and $\left.\varepsilon_{b}^{*}\right)$ we have used a linear interpolation between the GaAs and AlAs materials.

In Eq. (1) $V_{c}(z, P)$ is the potential that confines the donor electron in the well regions, which is given by

$$
V_{c}(z, P)= \begin{cases}\infty & \text { if }|z| \geq L_{c} \\ V_{0}(P) & \text { if }|z|<L_{b} / 2, \\ 0 & \text { if } L_{b} / 2<|z|<L_{c}\end{cases}
$$

where $L_{c}=L_{b} / 2+L_{w}$, with $L_{b}$ and $L_{w}$ being the hydrostaticpressure dependent widths of the central barrier and of a single $\mathrm{QW}$, respectively, obtained from the fractional change in the volume of the structure [11]. $V_{0}(P)$ is the pressure dependent barrier height. In order to include the pressure dependent $\Gamma-X$ mixing, we have followed the phenomenological model suggested by Elabsy [5].

The trial wave function for the ground state is chosen as $[6,7]$

$$
\Psi(r)=N f(z) g(r),
$$

where $N$ is a normalization constant, $g(r)=\exp (-\lambda r)$ is the hydrogenic part, where $\lambda$ is a variational parameter, and $f(z)$ is the eigenfunction of the Hamiltonian in Eq. (1) (without the impurity potential term), which is given by

$$
f(z)= \begin{cases}0 & \text { if }|z| \geq L_{c} \\ \cosh (\beta z) & \text { if }|z|<L_{b} / 2, \\ A[\sin (\eta z) & \text { if } L_{b} / 2<|z|<L_{c} \\ -\tan \left(\eta L_{c}\right) \cos (\eta z)\end{cases}
$$

where the constant $A$ is obtained from the continuity of the wave function. The corresponding eigenvalues associated with $f(z), E_{0 c}(P)$, may be obtained as the first root of the transcendental equation

$$
\begin{array}{r}
m_{w c}^{*} \beta \sin \left(\eta L_{w}\right) \sin \left(\beta L_{b} / 2\right) \\
-m_{b c}^{*} \eta \cos \left(\eta L_{w}\right) \cos \left(\beta L_{b} / 2\right)=0,
\end{array}
$$

where the constants $\beta$ and $\eta$ are defined by

$$
\eta=\sqrt{\left(2 m_{w c}^{*} / \hbar^{2}\right) E_{0 c}}
$$

and

$$
\beta=\sqrt{\left(2 m_{b c}^{*} / \hbar^{2}\right)\left(V_{0}-E_{0 c}\right)} .
$$

The hydrostatic pressure dependence of the donor binding energy is calculated as

$$
E_{b}(P)=E_{0 c}(P)-E_{\min }(P),
$$

where $E_{\min }(P)$ is the eigenvalue of the Hamiltonian in Eq. (1), minimized with respect to the variational parameter.

The transition probability per unit time for valence-todonor transitions (associated with a single impurity located at $z=z_{i}$ ) is proportional -in the dipole approximation- to the square of the matrix element of the electron-photon interaction between the wave functions of the initial (valence) and final (impurity) states, i.e.

$$
W=\frac{2 \pi}{\hbar} \sum_{i}\left|\left\langle f\left|H_{\mathrm{int}}\right| i\right\rangle\right|^{2} \delta\left(E_{f}-E_{i}-\hbar \omega\right)
$$

with $H_{\text {int }} \sim \vec{e} \cdot \vec{p}$, where $\vec{e}$ is the polarization vector in the direction of the electric field of the radiation and $\vec{p}$ is the momentum operator.

For a homogenous distribution of impurities, and assuming that the structure dimensions are much larger than the lattice spacing, the total transition probability $\left(W_{L}\right)$ is obtained by summing the contributions that come from all impurity positions [12]. The case of transitions from an acceptor-impurity band to the first conduction subband is obtained by performing the changes $m_{w c, b c}^{*} \leftrightarrow m_{w v, b v}^{*}$ and $V_{c}(z, P) \leftrightarrow V_{v}(z, P)$. For details of the calculations see for example the references $[11,12]$ and for the valence band used parameters, see Ref. [13].

\section{RESULTS AND DISCUSSION}

The hydrostatic pressure dependence of the DQW parameters is characterized by: (i) the electron (hole) conduction (valence) mass in the well and in the barrier increases linearly with pressure, (ii) the dielectric constant in the well and barrier decreases linearly with pressure, (iii) the volume change in the zinc-blend structure under hydrostatic pressure makes a linear reduction of the DQW widths, and finally (iv) the barrier height does not change in the range $0-13.5 \mathrm{kbar}$ and for higher pressure values it decreases up to zero at $P=33.5 \mathrm{kbar}$. All these effects contribute simultaneously, and in a competitive way, in the optical absorption processes that next we will discuss.


FIG. 1. Acceptor-impurity in a symmetrical GaAs- $\mathrm{Ga}_{0.7} \mathrm{Al}_{0.3} \mathrm{As}$ DQW with $L_{w}=50 \AA, L_{b}=0$, for $P=0$ (solid lines), $10 \mathrm{kbar}$ (dashed lines), and $30 \mathrm{kbar}$ (dotted lines). (a) binding energy as a function of the growth-direction impurity position. (b) optical-absorption spectra from the acceptor-impurity band to conduction ground state as function of the difference between the photon and gap energies.

Figure 1 shows the acceptor impurity band [Fig. 1(a)] for a DQW of dimensions $L_{w}=50 \AA$ and $L_{b}=0$, corresponding to a $L=100 \AA$ single $\mathrm{QW}$ as a proof that the DQW reproduces the results for single QW [12]. The three curves in order of increasing binding energy correspond to $P=0,10 \mathrm{kbar}$, and 
$30 \mathrm{kbar}$. It is important to point out that the $30 \mathrm{kbar}$ value is beyond the $\Gamma-X$ transition, which starts at $13.5 \mathrm{kbar}$, so the barrier height is smaller close to $40 \mathrm{meV}$ and the binding energy is larger as discussed in references [6, 7]. This is in agreement with the energy-pressure relation reported in $[6,7]$. Fig. 1(b) shows the optical absorption spectra related to transitions from the acceptor-impurity band to the conduction ground state. The energy displacement for the different pressure curves is larger in the absorption curve than in the binding energy curve due changes in the ground-state energy (both for valence and conduction states $E_{0 c}$ and $E_{0 v}$ ) as a function of pressure. The sharper peak in the absorption curves is due to the peak in the density of impurity states (DOIS) at $z_{i} / L_{c}= \pm 0.5$, which in this case is degenerate, while the shoulder structure is due to DOIS at $z_{i} / L_{c}=0$. It is noticeable an increasing red shift of the absorption spectra as the pressure increases essentially due to the pressure effects on the conduction ground state.
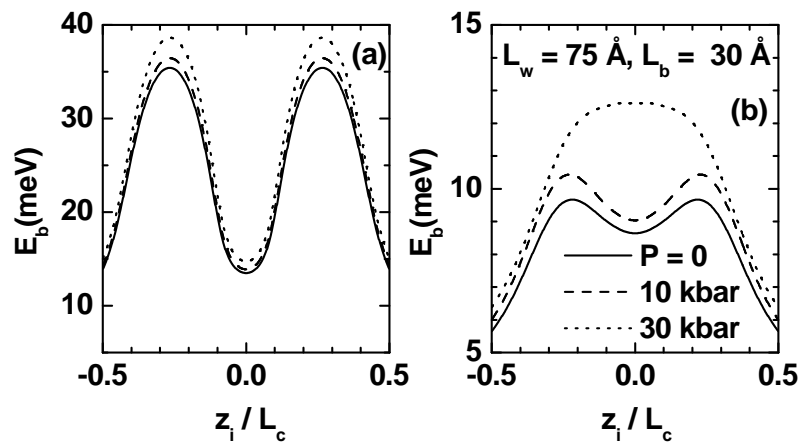

FIG. 2. Binding energy as function of the growth-direction impurity position in a symmetrical GaAs- $\mathrm{Ga}_{0.7} \mathrm{Al}_{0.3}$ As DQW with $L_{w}=75 \AA$, $L_{b}=30 \AA$, for $P=0$ (solid lines), $10 \mathrm{kbar}$ (dashed lines) and $30 \mathrm{kbar}$ (dotted lines). (a) acceptor-impurity, (b) donor-impurity.

Figure 2 shows the acceptor impurity band [Fig. 2(a)] and the donor impurity band [Fig. 2(b)] in a symmetrical GaAs$\mathrm{Ga}_{0.7} \mathrm{Al}_{0.3} \mathrm{As}$ DQW of dimensions $L_{w}=75 \AA$ and $L_{b}=30 \AA$. The three curves in each plot belong to $P=0,10 \mathrm{kbar}$, and 30 kbar. The acceptor impurity band shape, for the different showed pressures values, is quite analogous, presenting an energy increment as pressure increases. However, for the donor impurity band the shape at $P=30 \mathrm{kbar}$ is quit different from the other two, resembling that of a single QW. This is due to the indirect band gap regime discussed above where the central barrier is much lower in such a way, its effect on the two wells coupling is reduced, and the impurity electron cloud prefers to be close to $z_{i}=0$. As a consequence the binding energy is larger. The infinite barrier boundary conditions may contribute as well to this the effect, as can be seen from fig. 5 in reference [7]. There, the curve looks like the other two curves. Notice that for the other two pressure values the electron cloud is expelled from the central region of the structure due to the presence of the barrier. This effect is more pronounced for the hole case where they are more localized close to the well center. The reason of this behavior is the smaller effective Bohr radius so they are less confined by the structure and the central barrier is more effective in isolating the wells.

From the curves in Fig. 2(a) one observes that the corresponding DOIS will have two structures for the acceptor case, corresponding to the energies $(14 \mathrm{meV}$ for $P=0)$ at $z_{i} / L_{c}= \pm 0.5$ (triple degenerate) and at the center of the wells (35 meV for $P=0$, double degenerate). These two structures are clearly showed in Fig. 3(a) that corresponds to the DOIS for $P=30 \mathrm{kbar}$. In figure 3 (b) (which corresponds to the acceptor-impurity band to conduction ground state absorption) the two well defined structures are depicted as a shoulder, for $35 \mathrm{meV}$ at $P=0$, close to $1560 \mathrm{meV}$, and as a large peak close to $1580 \mathrm{meV}$. The same assignment is valid for the 30 kbar pressure values, the only difference being the pressure related blue shift associated with the pressure dependence of the GaAs band gap. Notice the line shape resemblance with the results of Fig. 1. The DOIS for the donor case [7] will have three structures, two peaks coming from the barrier center minimum, well center maximum; and a constant region coming from the lowest energies. The first two peaks are clearly seen in Fig. 3(c) for the optical absorption spectra from the valence ground state to the donor-impurity band. The third structure contributes to a high energy tail in the absorption spectra. The absorption process produces an overall blue shift, which overrules the pressure-related red shift discussed above, as pressure increases.
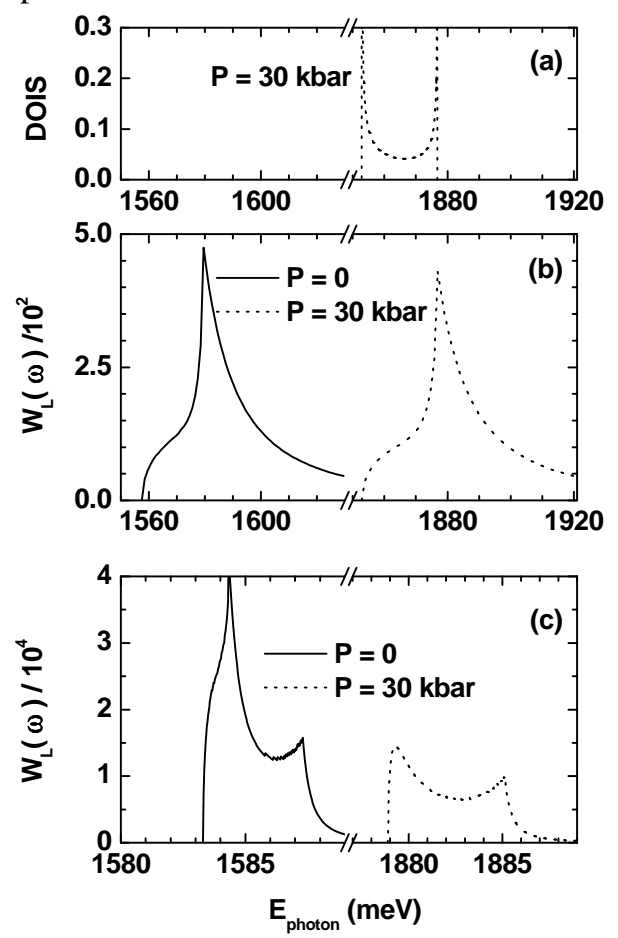

FIG. 3. Optical-absorption spectra and DOIS as a function of the photon energy in a symmetrical GaAs- $\mathrm{Ga}_{0.7} \mathrm{Al}_{0.3} \mathrm{As} \mathrm{DQW}$ with $L_{w}=75 \AA, L_{b}=30 \AA$, for $P=0$ (solid lines) and $30 \mathrm{kbar}$ (dotted lines). Figure 3(a) shows the DOIS corresponding to the shallowacceptor impurity band at $P=30$ kbar. Figure 3(b) corresponds to the absorption spectra from the acceptor-impurity band to the conduction ground state and 3(c) to the absorption spectra from the valence ground state to shallow-donor impurity band. 


\section{CONCLUSIONS}

By using the effective-mass approximation and the variational method, we have calculated the effects of hydrostatic pressure on the donor- and acceptor-related optical absorption spectra in symmetrical GaAs- $\mathrm{Ga}_{0.7} \mathrm{Al}_{0.3} \mathrm{As} \mathrm{DQW}$ structures. We have found that, depending of the sizes of the structure and the hydrostatic pressure, the donor-related absorption spectra will show two sharp structures, whereas for the acceptor case only one sharp peak is present while the other structure is very broad looking like a shoulder. This is due to sharper peaks in the DOIS for the donor case. These structures will appear in the absorption spectra in the case of a uniform distribution of impurities. Additionally, a red-shift in the optical transitions is observed with the increasing of the binding energy with the pressure, whereas a blue-shift is observed associated with the pressure dependence of the GaAs band gap. We expect that our work stimulate an experimental one on the pressure effects on the optical absorption spectra in GaAs- $\mathrm{Ga}_{1-x} \mathrm{Al}_{x} \mathrm{As}$ DQWs due to the fact that these structures could operate in the visible range and can be used for switching the output of the electronic devices.

\section{Acknowledgments}

We are grateful to the Universidad de Antioquia (CODI) for the financial support. This work was partially financed by the Colombian Scientific Agencies (Colciencias) under the grant 1115-05-11502.
[1] G. Bauer and T. Ando, Phys. Rev. B 38, 6015 (1998).

[2] M. Altarelli, U. Ekenberg, and A. Fasolino, Phys. Rev. B 32, 5138 (1985).

[3] D. Wolford and J. A. Bradley, Solid State Commun. 53, 1069 (1985).

[4] M. Holtz, M. Seon, O. Brafman, R. Manor, D. Fekete, Phys. Rev. B 54, 8714 (1996).

[5] A. M. Elabsy, J. Phys.: Condens. Matter 6, 10025 (1994).

[6] A. L. Morales, A. Montes, S. Y. López, and C. A. Duque, J. Phys.: Condens. Matter 14, 987 (2002); A. L. Morales, N. Raigoza, A. Montes, N. Porras-Montenegro, and C. A. Duque, Stat. Sol. (b) 241, 3224 (2004).
[7] N. Raigoza, A. L. Morales, A. Montes, N. Porras-Montenegro, and C. A. Duque, Phys. Rev. B 69, 045323 (2004).

[8] S. Adachi, J. Appl. Phys. 58, R1 (1985).

[9] G. A. Samara, Phys. Rev. B 27, 3494 (1983).

[10] R. F. Kopf, M. H. Herman, M. Lamont Schones, A. P. Perley, G. Livescu, and M. Ohring, J. Appl. Phys. 71, 5004 (1992).

[11] P. Y. Yu and M. Cardona, Fundamentals of Semiconductors (Springer-Verlag, Berlin, 1998).

[12] L. E. Oliveira and R. Pérez-Alvarez, Phys. Rev. B 40, 10460 (1989).

[13] E. Herbert Li, Physica E 5, 215 (2000). 